\title{
THE CHSH-TYPE INEQUALITIES FOR INFINITE-DIMENSIONAL QUANTUM SYSTEMS
}

\author{
YU GUO
}

\begin{abstract}
Aвstract. By establishing CHSH operators and CHSH-type inequalities, we show that any entangled pure state in infinite-dimensional systems is entangled in a $2 \otimes 2$ subspace. We find that, for infinite-dimensional systems, the corresponding properties are similar to that of the two-qubit case: (i) The CHSH-type inequalities provide a sufficient and necessary condition for separability of pure states; (ii) The CHSH operators satisfy the Cirel'son inequalities; (iii) Any state which violates one of these Bell inequalities is distillable.
\end{abstract}

\section{INTRODUCTION}

One of the entanglement problems is to decide whether or not a prepared state is entangled [1, 2, 3, 4, 5, 6, 7, 8, 9, 10]. The first condition of entanglement was given by Bell in 1964 [11]. Werner [12] first pointed out that separable states must satisfy all possible Bell inequalities. One of the most important Bell inequalities is the so-called CHSH inequalities investigated by Clauser, Horne, Shimony, and Holt [13] in 1969.

The CHSH inequalities have been studied intensively since they provide a way of experimentally testing the local hidden variable model as an independent hypothesis separated from the quantum formalism for two-qubit systems. In 1991, Gisin showed that the CHSH inequalities are not only necessary but also sufficient for separability of two-qubit pure states [14]. A short later, Gisin and Peres found that this fact can be extended into two-qudit systems [15]. And for the three-qubit case, Chen et al. proved that all pure entangled states violate some Bell inequality [16]. Since the Bell inequalities just provide a necessary condition for separability of mixed states, one might want to know which states violate the Bell inequalities. Consequently, it is verified that any two-qubit [17] or three-qubit [18] state violating a specific Bell inequality is distillable. This result also holds for $N$-qubit systems whenever the state is regarded as a bipartite state associated with the corresponding bipartite decomposition of the system [19, 20].

It is worth mentioning that the Bell inequalities can be used in verifying the security of quantum key distribution protocols [21] and that distillation of quantum entanglement plays a key role in quantum information processing [1]. In addition, one may even apply the CHSHtype inequality to one-body quantum system when investigating quantum contextuality [22].

PACS. 03.65.Db, 03.65.Ud, 03.67.Mn.

Key words and phrases. Entanglement; CHSH-type inequality; distillation; infinite-dimensional system. 
Very recently, M. Li et al. [23] proposed CHSH-type inequalities for any finite-dimensional composite system and showed that (i) a pure sate is entangled if and only if some of the $\mathrm{CHSH}$-type inequalities are violated and (ii) violating the $\mathrm{CHSH}$-type inequalities implies that the state is distillable. (Note here that Ref. [23] was originally devoted to extend the Gisin's Theorem to higher dimensional multipartite case, however, it did not complete the proof. Indeed it proposed CHSH-type inequalities, see Ref. [24] for detail.) Inspired by this, in this paper we discuss these issues for infinite-dimensional systems. (Infinite-dimensional quantum systems are always related to the continuous variable systems, such as harmonic oscillator, which has infinite eigenstates (or Fock states).)

This paper is organized as follows. In section 2 we construct the CHSH operators for infinite-dimensional systems. By virtue of the concurrence for infinite-dimensional systems [25](i.e., the concurrence of a pure state is zero if and only if it is separable), we can use an argument similar to that in Ref. [23] to prove the generalized CHSH-type inequalities. In addition, we find that the generalized CHSH operators also satisfy the Cirel'son inequalities. In section 3, a sufficient condition for distillation of entanglement in infinite-dimensional systems is proposed by reducing the given state to the two-qubit state and by the virtue of concurrence. Consequently, we conclude that any entangled pure sate in infinite-dimensional system is distillable. A final summary in the last section concludes this paper.

\section{The CHSH-TYPE INEQUALITIES FOR INFINITE-DIMENSIONAL COMPOSITE SYSTEMS}

Recall that, the CHSH operators for two-qubit quantum systems are constructed via the Pauli matrices, that is

$$
\mathcal{B}=A_{1} \otimes B_{1}+A_{1} \otimes B_{2}+A_{2} \otimes B_{1}-A_{2} \otimes B_{2},
$$

where $A_{i}=\overrightarrow{a_{i}} \cdot \overrightarrow{\sigma_{A}}=a_{i}^{x} \sigma_{A}^{x}+a_{i}^{y} \sigma_{A}^{y}+a_{i}^{z} \sigma_{A}^{z}, B_{j}=\overrightarrow{b_{j}} \cdot \overrightarrow{\sigma_{B}}=b_{j}^{x} \sigma_{B}^{x}+b_{j}^{y} \sigma_{B}^{y}+b_{j}^{z} \sigma_{B}^{z} ; \overrightarrow{a_{i}}=\left(a_{i}^{x}, a_{i}^{y}, a_{i}^{z}\right)$ and $\overrightarrow{b_{j}}=\left(b_{j}^{x}, b_{j}^{y}, b_{j}^{z}\right)$ are real vectors satisfying $\left(a_{i}^{x}\right)^{2}+\left(a_{i}^{y}\right)^{2}+\left(a_{i}^{z}\right)^{2}=1,\left(b_{j}^{x}\right)^{2}+\left(b_{j}^{y}\right)^{2}+\left(b_{j}^{z}\right)^{2}=1$; $\sigma_{A / B}^{x, y, z}$ are Pauli matrices. The CHSH-type inequalities read as

$$
|\langle\mathcal{B}\rangle| \leq 2 .
$$

Namely, if there exist local hidden variable models describing the system, then the inequalities in (2.2) must hold.

In the following, we construct $\mathrm{CHSH}$ operators for infinite-dimensional quantum systems.

2.1. Bipartite systems. Consider a system consisting of two parts, labeled A and B, with state spaces $H_{A}$ and $H_{B}\left(\operatorname{dim} H_{A} \otimes H_{B}=+\infty\right)$ respectively. For the fixed basis $\{|i\rangle\}_{i=1}^{+\infty}$ of $H_{A}$, let $L_{1}^{A}=|1\rangle\langle 2|-| 2\rangle\left\langle 1\left|, L_{2}^{A}=\right| 1\right\rangle\langle 3|-| 3\rangle\left\langle 1\left|, L_{3}^{A}=\right| 2\right\rangle\langle 3|-| 3\rangle\left\langle 2\left|, L_{4}^{A}=\right| 1\right\rangle\langle 4|-| 4\rangle\langle 1|$, $L_{5}^{A}=|2\rangle\langle 4|-| 4\rangle\left\langle 2\left|, L_{6}^{A}=\right| 3\right\rangle\langle 4|-| 4\rangle\left\langle 3\left|, L_{7}^{A}=\right| 1\right\rangle\langle 5|-| 5\rangle\langle 1|, \cdots$, and $L_{\alpha}^{A}=|i\rangle\langle j|-| j\rangle\langle i|$ with $\alpha=(j-2)$ ! $+i$ whenever $j \geq 5$. Similarly, we can define $\left\{L_{\beta}^{B}\right\}$. The operators $L_{\alpha}^{A}$ (resp. $\left.L_{\beta}^{B}\right)$ 
have two none zero entries. We define $A_{i}^{\alpha}\left(\operatorname{resp} . B_{j}^{\beta}\right.$ ) from $L_{\alpha}^{A}\left(\operatorname{resp} . L_{\beta}^{B}\right)$ by replacing the four entries on the positions of the nonzero two rows and two columns of $L_{\alpha}^{A}$ (resp. $L_{\beta}^{B}$ ) with the corresponding four entries of the matrix $\overrightarrow{a_{i}} \overrightarrow{\sigma_{A}}$ (resp. $\overrightarrow{b_{j}} \overrightarrow{\sigma_{B}}$ ), and keeping the other entries of $A_{i}^{\alpha}\left(\right.$ resp. $B_{j}^{\beta}$ ) zero. We define the CHSH operators to be

$$
\mathcal{B}_{\alpha \beta}=\tilde{A}_{1}^{\alpha} \otimes \tilde{B}_{1}^{\beta}+\tilde{A}_{1}^{\alpha} \otimes \tilde{B}_{2}^{\beta}+\tilde{A}_{2}^{\alpha} \otimes \tilde{B}_{1}^{\beta}-\tilde{A}_{2}^{\alpha} \otimes \tilde{B}_{2}^{\beta}
$$

where $\tilde{A}_{i}^{\alpha}=L_{\alpha}^{A} A_{i}^{\alpha}\left(L_{\alpha}^{A}\right)^{\dagger}, \tilde{B}_{j}^{\beta}=L_{\beta}^{B} B_{j}^{\beta}\left(L_{\beta}^{B}\right)^{\dagger}, i, j=1,2$.

Let us extend this idea to the multipartite case in the following.

2.2. Multipartite systems. In this subsection, we discuss the multipartite system $H_{1} \otimes H_{2} \otimes$ $\cdots \otimes H_{m}, \operatorname{dim} H_{1} \otimes H_{2} \otimes \cdots \otimes H_{m}=+\infty$. Any pure state $|\psi\rangle \in H_{1} \otimes H_{2} \otimes \cdots \otimes H_{m}$ can be represented by

$$
|\psi\rangle=\sum_{i_{1}, i_{2}, \cdots, i_{m}} a_{i_{1} i_{2} \cdots i_{m}}\left|i_{1} i_{2} \cdots i_{m}\right\rangle
$$

Let $\alpha$ and $\alpha^{\prime}$ (respectively, $\beta$ and $\beta^{\prime}$ ) be subsets of the sub-indices of $a$, associated to the same sub-vector spaces but with different summing indices. $\alpha$ (or $\alpha^{\prime}$ ) and $\beta$ (or $\beta^{\prime}$ ) span the whole space of the given sub-index of $a$. Possible combinations of the indices of $\alpha$ and $\beta$ can be equivalently understood as a kind of bipartite decomposition of the $m$ subsystems, say part $\mathrm{A}^{\prime}$ and part $\mathrm{B}^{\prime}$, containing $s$ and $t=m-s$ subsystems respectively.

For a fixed bipartite decomposition, the system can be regarded as a bipartite system. Let $L_{\alpha}^{A}$ and $L_{\beta}^{B}$ be the operators defined as above. Let $\rho=|\psi\rangle\langle\psi|$, then

$$
\rho_{\alpha \beta}^{p}=\frac{L_{\alpha}^{A} \otimes L_{\beta}^{B} \rho\left(L_{\alpha}^{A}\right)^{\dagger} \otimes\left(L_{\beta}^{B}\right)^{\dagger}}{\left\|L_{\alpha}^{A} \otimes L_{\beta}^{B} \rho\left(L_{\alpha}^{A}\right)^{\dagger} \otimes\left(L_{\beta}^{B}\right)^{\dagger}\right\|_{T r}}
$$

is a "two-qubit" pure state, where $p$ denotes the fixed bipartite decomposition. Now, for any given bipartite decomposition, we can define the corresponding $\mathrm{CHSH}$ operators

$$
\mathcal{B}_{\alpha \beta}^{p}=\tilde{A}_{1}^{\alpha} \otimes \tilde{B}_{1}^{\beta}+\tilde{A}_{1}^{\alpha} \otimes \tilde{B}_{2}^{\beta}+\tilde{A}_{2}^{\alpha} \otimes \tilde{B}_{1}^{\beta}-\tilde{A}_{2}^{\alpha} \otimes \tilde{B}_{2}^{\beta},
$$

where $\tilde{A}_{i}^{\alpha}=L_{\alpha}^{A} A_{i}^{\alpha}\left(L_{\alpha}^{A}\right)^{\dagger}$ and $\tilde{B}_{j}^{\beta}=L_{\beta}^{B} B_{j}^{\beta}\left(L_{\beta}^{B}\right)^{\dagger}$ are the self-adjoint operators defined as in Eq.(2.3).

By the feature of concurrence, M. Li et al. generalized the CHSH operators to arbitrary finite-dimensional cases [23]. Very recently, we proposed concurrence for infinitedimensional cases:

Definition 2.1.(Guo et al. [25]) Let $|\psi\rangle \in H_{A} \otimes H_{B}$ be a pure state with $\operatorname{dim} H_{A} \otimes H_{B}=+\infty$, and let $\{|i\rangle\}$ and $\{|j\rangle\}$ be the bases of $H_{A}$ and $H_{B}$, respectively. Write $|\psi\rangle=\sum_{i, j} a_{i j}|i\rangle|j\rangle$. Then

$$
C(|\psi\rangle)=\sqrt{2\left(1-\operatorname{Tr}\left(\rho_{A}^{2}\right)\right)}, \rho_{A}=\operatorname{Tr}_{B}(|\psi\rangle\langle\psi|)
$$


is called the concurrence of $|\psi\rangle$. Moreover,

$$
C(|\psi\rangle)=\sqrt{\sum_{i, j, k, l}\left|a_{i k} a_{j l}-a_{i l} a_{j k}\right|^{2}}
$$

It is clear that $C(|\psi\rangle)=0$ if and only if $|\psi\rangle$ is separable. Letting $\rho_{\alpha \beta}=\frac{L_{\alpha}^{A} \otimes L_{\beta}^{B} \rho\left(L_{\alpha}^{A}\right)^{\dagger} \otimes\left(L_{\beta}^{B}\right)^{\dagger}}{\left\|L_{\alpha}^{A} \otimes L_{\beta}^{B} \rho\left(L_{\alpha}^{A}\right)^{\dagger} \otimes\left(L_{\beta}^{B}\right)^{\dagger}\right\|_{\mathrm{Tr}}}$, one can check that

$$
C(|\psi\rangle)=\sqrt{\sum_{\alpha} \sum_{\beta}\left[C\left(\rho_{\alpha \beta}\right)\right]^{2}} .
$$

With this basic idea in mind, similar to that of the finite-dimensional case [26],we define concurrence for infinite-dimensional multipartite systems.

Definition 2.2. Let $\left\{\left|i_{k}\right\rangle\right\}$ be the basis of $H_{k}, k=1,2, \ldots, m,|\psi\rangle=\sum_{i_{1}, i_{2}, \cdots, i_{m}} a_{i_{1} i_{2} \cdots i_{m}}\left|i_{1} i_{2} \cdots i_{m}\right\rangle \in$ $H_{1} \otimes H_{2} \otimes \cdots \otimes H_{m}$ be a pure state with $\operatorname{dim} H_{1} \otimes H_{2} \otimes \cdots \otimes H_{m}=+\infty$. Then

$$
C(|\psi\rangle)=\sqrt{\frac{1}{2^{m-1}-1} \sum_{p} \sum_{\alpha, \alpha^{\prime}, \beta, \beta^{\prime}}\left|a_{\alpha \beta} a_{\alpha^{\prime} \beta^{\prime}}-a_{\alpha \beta^{\prime}} a_{\alpha^{\prime} \beta}\right|^{2}}
$$

is called the concurrence of $|\psi\rangle$. Here $\alpha$ and $\alpha^{\prime}$ (respectively, $\beta$ and $\beta^{\prime}$ ) are subsets of the subindices of $a$, associated to the same sub Hilbert space but with different summing indices; $\alpha$ (or $\alpha^{\prime}$ ) and $\beta$ (or $\beta^{\prime}$ ) span the whole space of a given subindex of $a$; and where $\sum_{p}$ stands for the summation over all possible combinations of the indices of $\alpha$ and $\beta$.

From Definition 2.2, one can obtain that $C(|\psi\rangle)=0$ if and only if $|\psi\rangle$ is fully separable (namely, $|\psi\rangle=\left|\psi_{1}\right\rangle \otimes\left|\psi_{2}\right\rangle \otimes \cdots \otimes\left|\psi_{m}\right\rangle$ ). It is easy to see that

$$
C(|\psi\rangle)=\sqrt{\frac{1}{2^{m-1}-1} \sum_{p} \sum_{\alpha, \beta}\left[C\left(\rho_{\alpha \beta}^{p}\right)\right]^{2}} .
$$

Based on Eq.(2.6) and Eq.(2.8), using an argument similar to that in Ref. [23] one can obtain the following results.

Theorem 2.3. Let $\rho$ be a bipartite pure state acting on $H_{A} \otimes H_{B}$ with $\operatorname{dim} H_{A} \otimes H_{B}=+\infty$. Then $\rho$ is separable if and only if $\rho$ satisfies all Bell inequalities, i.e.,

$$
\left|\left\langle\mathcal{B}_{\alpha \beta}\right\rangle\right| \leq 2
$$

for all CHSH operators as in Eq.(2.3).

Theorem 2.4. Let $\rho$ be a multipartite pure state acting on $H_{1} \otimes H_{2} \otimes \cdots \otimes H_{m}$ with $\operatorname{dim} H_{1} \otimes$ $H_{2} \otimes \cdots \otimes H_{m}=+\infty$. Then $\rho$ is separable if and only if $\rho$ satisfies all Bell inequalities, i.e.,

$$
\left|\left\langle\mathcal{B}_{\alpha \beta}^{p}\right\rangle\right| \leq 2
$$


for all CHSH operators as in Eq.(2.4).

Let $\mathcal{S}_{s-p}$ be the set of all separable pure states. Observe that, any separable state $\rho$ acting on $H_{A} \otimes H_{B}$ admits a representation of the Bochner integral [27]

$$
\rho=\int_{\mathcal{S}_{s-p}} \varphi\left(\rho^{A} \otimes \rho^{B}\right) d \mu\left(\rho^{A} \otimes \rho^{B}\right),
$$

where $\mu$ is a Borel probability measure on $\mathcal{S}_{s-p}, \rho^{A} \otimes \rho^{B} \in \mathcal{S}_{s-p}$, and $\varphi: \mathcal{S}_{s-p} \rightarrow \mathcal{S}_{s-p}$ is a measurable function. (Note that the above definition is equivalent to that given in [12, 28].) Thus, for any $\mathrm{CHSH}$ operator $\mathcal{B}_{\alpha \beta}$, it is straightforward that

$$
\begin{aligned}
\left|\left\langle\mathcal{B}_{\alpha \beta}\right\rangle\right| & \leq \int_{\mathcal{S}_{s-p}}\left|\operatorname{Tr}\left[\varphi\left(\rho^{A} \otimes \rho^{B}\right) \mathcal{B}_{\alpha \beta}\right]\right| d \mu\left(\rho^{A} \otimes \rho^{B}\right) \\
& \leq 2
\end{aligned}
$$

That is, all separable mixed states also satisfy the inequalities in (2.9). Similarly, all fully separable multipartite mixed states satisfying the inequalities in (2.10).

Going further, we discuss the relation between the $\mathrm{CHSH}$ operators and entanglement witnesses. It is shown in [29] that a given state is entangled if and only if there exists at least one entanglement witness detecting it. An entanglement witness $W$ is a self-adjoint operator (also sometimes called a Hermitian operator) acting on $H_{A} \otimes H_{B}$ that satisfies $\operatorname{Tr}(W \sigma) \geq 0$ for all separable sates $\sigma$ and $\operatorname{Tr}(W \rho)<0$ for at least one entangled state $\rho$. Now, letting

$$
W_{\alpha \beta}=2 I-\mathcal{B}_{\alpha \beta}
$$

it is clear that $\operatorname{Tr}(W \sigma) \geq 0$ for all separable states and $\operatorname{Tr}(W \rho)<0$ if and only if $\left|\left\langle\mathcal{B}_{\alpha \beta}\right\rangle\right| \leq 2$. Thus, $W_{\alpha \beta}$ is an entanglement witness provided that $2 I-\mathcal{B}_{\alpha \beta}$ is not positive. Form this point of view, the $\mathrm{CHSH}$ operators are entanglement witnesses that can detect all entangled pure states.

For two-qubit systems, the CHSH operators satisfy the Cirel'son inequality [30]

$$
|\langle\mathcal{B}\rangle| \leq 2 \sqrt{2}
$$

for all states $\rho$ and all CHSH operators $\mathcal{B}$. The state $\rho=\frac{1}{2}(|01\rangle-|10\rangle)(\langle 01|-\langle 10|)$ saturates the Cirel'son bound, i.e., $|\operatorname{Tr}(\mathcal{B} \rho)|=2 \sqrt{2}$.

Theorem 2.5. The Cirel'son inequality holds for composite systems of any dimension.

Proof. Now we consider the generalized CHSH operators (for both finite- and infinite-dimensional cases). We write $P_{\alpha}=|i\rangle\langle i|+| j\rangle\langle j|$ whenever $L_{\alpha}=|i\rangle\langle j|-| j\rangle\langle i|$ and define $P_{\beta}$ similarly. Then we consider the squares of $\tilde{A}_{i}^{\alpha}$ and $\tilde{B}_{j}^{\beta}:\left(\tilde{A_{i}^{\alpha}}\right)^{2}=L_{\alpha} A_{i}^{\alpha} L_{\alpha}^{\dagger} L_{\alpha} A_{i}^{\alpha} L_{\alpha}^{\dagger}=L_{\alpha}\left(A_{i}^{\alpha}\right)^{2} L_{\alpha}^{\dagger}=P_{\alpha}$, 
$\left(\tilde{B}_{j}^{\beta}\right)^{2}=P_{\beta}$. Using the fact

$$
\begin{aligned}
& \tilde{A}_{1}^{\alpha} \otimes \tilde{B}_{1}^{\beta}+\tilde{A}_{1}^{\alpha} \otimes \tilde{B}_{2}^{\beta}+\tilde{A}_{2}^{\alpha} \otimes \tilde{B}_{1}^{\beta}-\tilde{A}_{2}^{\alpha} \otimes \tilde{B}_{2}^{\beta} \\
= & \frac{1}{\sqrt{2}}\left(\left(\tilde{A}_{1}^{\alpha}\right)^{2} \otimes P_{\beta}+\left(\tilde{A}_{2}^{\alpha}\right)^{2} \otimes P_{\beta}\right. \\
& \left.+P_{\alpha} \otimes\left(\tilde{B}_{1}^{\beta}\right)^{2}+P_{\alpha} \otimes\left(\tilde{B}_{2}^{\beta}\right)^{2}\right) \\
& -\frac{\sqrt{2}-1}{8}\left((\sqrt{2}+1)\left(\tilde{A}_{1}^{\alpha} \otimes P_{\beta}-P_{\alpha} \otimes \tilde{B}_{1}^{\beta}\right)\right. \\
& \left.+\tilde{A}_{2}^{\alpha} \otimes P_{\beta}-P_{\alpha} \otimes \tilde{B}_{2}^{\beta}\right)^{2} \\
& -\frac{\sqrt{2}-1}{8}\left((\sqrt{2}+1)\left(\tilde{A}_{1}^{\alpha} \otimes P_{\beta}-P_{\alpha} \otimes \tilde{B}_{2}^{\beta}\right)\right. \\
& \left.-\tilde{A}_{2}^{\alpha} \otimes P_{\beta}-P_{\alpha} \otimes \tilde{B}_{1}^{\beta}\right)^{2} \\
& -\frac{\sqrt{2}-1}{8}\left((\sqrt{2}+1)\left(\tilde{A}_{2}^{\alpha} \otimes P_{\beta}-P_{\alpha} \otimes \tilde{B}_{1}^{\beta}\right)\right. \\
& \left.+\tilde{A}_{1}^{\alpha} \otimes P_{\beta}+P_{\alpha} \otimes \tilde{B}_{2}^{\beta}\right)^{2} \\
& -\frac{\sqrt{2}-1}{8}\left((\sqrt{2}+1)\left(\tilde{A}_{2}^{\alpha} \otimes P_{\beta}+P_{\alpha} \otimes \tilde{B}_{1}^{\beta}\right)\right. \\
& \left.-\tilde{A}_{1}^{\alpha} \otimes P_{\beta}-P_{\alpha} \otimes \tilde{B}_{1}^{\beta}\right)^{2} \\
\leq & \frac{1}{\sqrt{2}}\left(\left(\tilde{A}_{1}^{\alpha}\right)^{2} \otimes P_{\beta}\right)+\left(\tilde{A}_{2}^{\alpha}\right)^{2} \otimes P_{\beta} \\
& \left.+P_{\alpha} \otimes\left(\tilde{B}_{1}^{\beta}\right)^{2}+P_{\alpha} \otimes\left(\tilde{B}_{2}^{\beta}\right)^{2}\right) \\
= & 2 \sqrt{2} P_{\alpha} \otimes P_{\beta}
\end{aligned}
$$

we have

$$
\left|\left\langle\mathcal{B}_{\alpha \beta}\right\rangle\right| \leq 2 \sqrt{2}
$$

since $\left|\left\langle P_{\alpha} \otimes P_{\beta}\right\rangle\right| \leq 1$. Similarly, we have

$$
\left|\left\langle\mathcal{B}_{\alpha \beta}^{p}\right\rangle\right| \leq 2 \sqrt{2}
$$

\section{CHSH-TYPE INEQUALITIES AND DISTILLATION}

For the finite-dimensional case, it is shown in Ref. [23] that quantum states that violate the CHSH-type inequalities must be distillable. In this section, we discuss the infinitedimensional case.

The famous singlet states play a crucial role in quantum information theory since for many tasks, like teleportation and cryptography, one ideally needs maximally entangled two-qubit states. However, in laboratories we usually have mixed states due to imperfection of operations and decoherence. It makes sense to transform the mixed states to the useful singlet states. Recall that, a state $\rho$ is defined to be distillable if and only if the singlet state (e.g. $\left.\left|\psi^{ \pm}\right\rangle=\frac{1}{\sqrt{2}}(|00\rangle \pm|11\rangle)\right)$ can be obtained from a finite number of copies of $\rho$ by LOCC. In Ref. [23], in the finite-dimensional multipartite system, a multipartite state is called distillable if and only if there exists some bipartite decomposition of the system such that the state is distillable whenever it is regarded as a bipartite state. 
Any LOCC for an infinite-dimensional bipartite system admits the form of

$$
\Lambda(\rho)=\sum_{i=1}^{N} A_{i} \otimes B_{i} \rho A_{i}^{\dagger} \otimes B_{i}^{\dagger}
$$

where $A_{i}$ and $B_{i}$ are operators acting on $H_{A}$ and $H_{B}$ respectively, $\sum_{i=1}^{N} A_{i}^{\dagger} A_{i} \otimes B_{i}^{\dagger} B_{i}=I_{A} \otimes I_{B}$, and the series converges in the strong operator topology [7] and $N$ may be $+\infty$.

For an infinite-dimensional multipartite system, we can define distillation of entanglement as follows.

Definition 3.1. Let $H_{i}, i=1,2, \ldots, m$, be complex separable Hilbert spaces with $\operatorname{dim} H_{1} \otimes$ $H_{2} \otimes \cdots \otimes H_{m}=+\infty$. A state $\rho$ acting on $H_{1} \otimes H_{2} \otimes \cdots \otimes H_{m}$ is said to be distillable if and only if there exists some bipartite decomposition of the system such that $\rho$ is distillable whenever it is regarded as a bipartite state $\check{\rho}=\rho^{A^{\prime} B^{\prime}}$ (acting on $H_{A^{\prime}} \otimes H_{B^{\prime}}=H_{1^{\prime}} \otimes H_{2^{\prime}} \otimes \cdots \otimes H_{m^{\prime}}$, where $\left.\left\{1^{\prime}, 2^{\prime}, \ldots, m^{\prime}\right\}=\{1,2, \ldots, m\}\right)$, i.e., $\rho$ is distillable if and only if there exists some LOCC $\Lambda$ and a finite number $n$ such that

$$
\Lambda\left(\check{\rho}^{\otimes n}\right) \text { is a singlet state. }
$$

It is obvious that a bipartite state $\rho$ is distillable if there exist some projectors $P$ and $Q$ that map $H_{A}$ and $H_{B}$ into $\mathbb{C}^{2}$ and $\mathbb{C}^{2}$ respectively such that $P \otimes Q \rho P \otimes Q$ is entangled.

The main result of this section is the following.

Theorem 3.2. Let $H_{A}$ and $H_{B}$ be complex separable Hilbert spaces with $\operatorname{dim} H_{A} \otimes H_{B}=+\infty$. If the state $\rho$ acting on $H_{A} \otimes H_{B}$ violates one of the CHSH-type inequalities in (2.9), then $\rho$ is distillable. Moreover, assume that $\operatorname{dim} H_{1} \otimes H_{2} \otimes \cdots \otimes H_{m}=+\infty$, if the state $\rho$ acting on $H_{1} \otimes H_{2} \otimes \cdots \otimes H_{m}$ violate one of the CHSH-type inequalities in (2.10), then $\rho$ is distillable.

Proof. We only need to prove the first part; the multipartite case can be checked similarly.

If $\operatorname{Tr}\left(B_{\alpha_{0} \beta_{0}} \rho\right)>2$ for some $\alpha_{0}$ and $\beta_{0}$, then

$$
\rho_{\alpha_{0} \beta_{0}}=\frac{L_{\alpha_{0}}^{A} \otimes L_{\beta_{0}}^{B} \rho\left(L_{\alpha_{0}}^{A}\right)^{\dagger} \otimes\left(L_{\beta_{0}}^{B}\right)^{\dagger}}{\left\|L_{\alpha_{0}}^{A} \otimes L_{\beta_{0}}^{B} \rho\left(L_{\alpha_{0}}^{A}\right)^{\dagger} \otimes\left(L_{\beta_{0}}^{B}\right)^{\dagger}\right\|_{\mathrm{Tr}}}
$$

and $C\left(\rho_{\alpha_{0} \beta_{0}}\right)>0$. Write $L_{\alpha_{0}}=|i\rangle\langle j|-| j\rangle\left\langle i\left|, L_{\beta_{0}}=\right| k\right\rangle\langle l|-| l\rangle\langle k|$. Let $P=A L_{\alpha_{0}}$ and $Q=B L_{\beta_{0}}$ with $A=\left|0_{A}\right\rangle\left\langle i|+| 1_{A}\right\rangle\left\langle j|, B=| 0_{B}\right\rangle\left\langle k|+| 1_{B}\right\rangle\langle l|$, where $\left|0_{A, B}\right\rangle$ and $\left|1_{A, B}\right\rangle$ are orthonormal bases of $\mathbb{C}^{2}$. It is clear that $P \otimes Q \rho P \otimes Q$ is a two-qubit state and $C(P \otimes Q \rho P \otimes Q)=C\left(\rho_{\alpha_{0} \beta_{0}}\right)$. Therefore $\rho$ is distillable since any two-qubit state is distillable.

Like the finite-dimensional case, the infinite-dimensional PPT state should never violate the CHSH-type inequalities in (2.9) or (2.10) since PPT states are not distillable. In the other 
words, the CHSH operators are entanglement witnesses that cannot detect any PPT entangled state. In Ref. [31], it is shown that any entangled pure state in a finite-dimensional system is distillable. Together with Theorem 3.2, we can conclude that:

Proposition 3.3. Let $H_{A}$ and $H_{B}$ be complex separable Hilbert spaces with $\operatorname{dim} H_{A} \otimes H_{B} \leq$ $+\infty$, and let $|\psi\rangle \in H_{A} \otimes H_{B}$ be a pure state. Then $|\psi\rangle$ is distillable if and only if it is entangled.

Namely, there are no bound entangled pure states in either finite- or infinite-dimensional systems.

\section{Conclusion}

In summary, the CHSH-type inequalities for the infinite-dimensional systems were proposed. We showed that, in nature, for any entangled pure state, there exists at least one $2 \otimes 2$ subspace such that the associated state is entangled and therefore the corresponding properties are valid. (Note that, for mixed states, Sperling and Vogel showed in Ref. [32] that any bipartite entanglement in infinite-dimensional systems can be identified in finite-dimensional Hilbert spaces, even though in general the reduction in the Hilbert space to finite dimensions may lead to nonclassical artifacts.) The generalized CHSH-type inequalities may help in the measurable determination of quantum entanglement experimentally since there is a one-toone correspondence between the CHSH operators and the entanglement witnesses.

Acknowledgments The author wishes to thank the referees for their useful comments and suggestions. This work is supported by the China Postdoctoral Science Foundation funded project (2012M520603), the National Natural Science Foundation of China (11171249, 11101250), the Natural Science Foundation of Shanxi (2013021001-1, 2012011001-2), the Research start-up fund for Doctors of Shanxi Datong University (2011-B-01) and the Scientific and Technological Innovation Programs of Higher Education Institutions in Shanxi (20121015).

\section{REFERENCES}

[1] M. A. Nielsen, I. L. Chuang, Quantum Computatation and Quantum Information (Cambridge University Press, Cambridge, 2000).

[2] R. Horodecki, P. Horodecki, M. Horodecki, and K. Horodecki, Rev. Mod. Phys. 81 (2009) 865.

[3] O. Gühne and G. Tóth, Phys. Rep. 474 (2009) 1.

[4] X. Zhao, H. Wu, Y. S. Li and G. L. Long, Chin. Phys. Lett. 26(6) (2009) 060305.

[5] D. H. Zhang, D. L. Zhou and H. Fan, Chin. Phys. Lett. 27(9) 090306 (2010).

[6] Y. Wang, J. Hou and Y. Guo, Chin. Sci. Bull. 57(14) (2012) 1643-1647.

[7] J. Hou, J. Phys A: Math. Theor. 43 (2010) 385201.

[8] J. Hou and Y. Guo, Phys. Rev. A 82 (2010) 052301.

[9] Y. Guo and J. Hou, J. Phys. A: Math. Theor. 45 (2012) 505303. 
[10] Y. Guo, X. Qi and J. Hou, Chin. Sci. Bull. 56(9) (2011) 840-846.

[11] J. S. Bell, Physcs (Long Island City, N.Y.) 1 (1964) 195.

[12] R. F. Werner, Phys. Rev. A 40 (1989) 4277.

[13] J. F. Clauser, M. A. Horne et al, Phys. Rev. Lett. 23 (1969) 880.

[14] N. Gisin, Phys. Lett. A 154 (1991) 201.

[15] N. Gisin and A. Peres, Phys. Lett. A 162 (1992) 15.

[16] J. L. Chen, C. F. Wu, L. C. Kwek and C. H. Oh, Phys. Rev. Lett. 93 (2004) 140407.

[17] R. Horodecki, M. Horodecki, P. Horodecki, Phys. Lett. A 222 (1996) 21-25.

[18] S. Lee, J. Joo and J. Kim, Phys. Rev. A 76 (2007) 012311.

[19] A. Acín, Phys. Rev. Lett. 88 (2002) 027901.

[20] A. Acín, V. Scarani and M. M. Wolf, Phys. Rev. A, 66 (2002) 042323.

[21] A. K. Ekert, Phys. Rev. Lett. 67(6) (1991) 661-663; A. Acín et al. Phys. Rev. Lett. 98 (2007) 230501.

[22] H.-Y. Su, J.-L. Chen, C. Wu, S Yu and C. H. Oh, Phys. Rev. A 85 (2012) 052126; J.-L. Chen, H.-Y. Su1, C. Wu, D.-L. Deng, A. Cabello, L. C. Kwek and C. H. Oh, Phys. Rev. A 87 (2013) 022109.

[23] M. Li and S. M. Fei, Phys. Rev. Lett. 104 (2010) 240502.

[24] S. K. Choudhary, S. Ghosh, G. Kar and R. Rahaman, Phys. Rev. Lett. 105 (2010) 218901; S. Yu, Q. Chen, C. Zhang, C. H. Lai and C. H. Oh, Phys. Rev. Lett. 109 (2010) 120402; J.-L. Chen, C. Wu, L. C. Kwek and C. H. Oh, Phys. Rev. A 78 (2008) 032107; J.-L. Chen, D.-L. Deng and M.-G. Hu, Phys. Rev. A 77 (2008) 060306(R); C. Wu, J.-L. Chen, L. C. Kwek and C. H. Oh, Phys. Rev. A 77 (2008) 062309.

[25] Y. Guo, J. Hou and Y. Wang, arXiv:1203.3933v1.

[26] S. Albeverio and S. M. Fei, J. Opt. B: Quant. Semiclass. Opt. 3 (2001) 223-227.

[27] A. S. Holevo, M. E. Shirokov and R. F. Werner, Russ. Math. Surv. 60 (2005) 153-154.

[28] P. Horodecki and R. Horodecki, Quant. Inf. comput. 1(1) (2001) 45-75.

[29] M. Horodecki, P. Horodecki and R. Horodecki, Phys. Lett. A 223 (1996) 1-8.

[30] B. S. Cirelson, Lett. Math. Phys. 4 (1980) 93-100.

[31] C. H. Bennett, H. J. Bernstein, S. Popescu and B. Schumacher, Phys. Rev. A 53 (1996) 2046.

[32] J. Sperling and W. Vogel, Phys. Rev. A 79 (2009) 052313.

Department of Mathematics, Shanxi Datong University, Datong, 037009, China

E-mail address: guoyu3@aliyun.com; yuguophys@aliyun.com 